\title{
Spatiotemporal integration for tactile localization during arm movements: a
}

\section{probabilistic approach}

\author{
Femke Maij, ${ }^{1,2}$ Alan M. Wing, ${ }^{2}$ and W. Pieter Medendorp ${ }^{1}$ \\ ${ }^{1}$ Radboud University Nijmegen, Donders Institute for Brain, Cognition and Behaviour, Nijmegen, The Netherlands; \\ and ${ }^{2}$ School of Psychology, University of Birmingham, Edgbaston, Birmingham, United Kingdom
}

Submitted 7 November 2012; accepted in final form 21 August 2013

\begin{abstract}
Maij F, Wing AM, Medendorp WP. Spatiotemporal integration for tactile localization during arm movements: a probabilistic approach. J Neurophysiol 110: 2661-2669, 2013. First published August 21, 2013; doi:10.1152/jn.00971.2012.- It has been shown that people make systematic errors in the localization of a brief tactile stimulus that is delivered to the index finger while they are making an arm movement. Here we modeled these spatial errors with a probabilistic approach, assuming that they follow from temporal uncertainty about the occurrence of the stimulus. In the model, this temporal uncertainty converts into a spatial likelihood about the external stimulus location, depending on arm velocity. We tested the prediction of the model that the localization errors depend on arm velocity. Participants $(n=8)$ were instructed to localize a tactile stimulus that was presented to their index finger while they were making either slow- or fast-targeted arm movements. Our results confirm the model's prediction that participants make larger localization errors when making faster arm movements. The model, which was used to fit the errors for both slow and fast arm movements simultaneously, accounted very well for all the characteristics of these data with temporal uncertainty in stimulus processing as the only free parameter. We conclude that spatial errors in dynamic tactile perception stem from the temporal precision with which tactile inputs are processed.
\end{abstract}

haptics; mislocalization; human; movement; perception

TACTILE STIMULI, e.g., a touch stimulus applied to the arm, are initially encoded in a somatotopic reference frame. To localize these tactile stimuli in external space a coordinate transformation is required, which must take postural information about the arm into account. Previous behavioral and neurophysiological studies have shown that this transformation process takes time (Azanon and Soto-Faraco 2008; Heed and Roder 2010).

Under normal conditions the arm is typically not stationary at the time of a tactile stimulus. This provides additional complexity to tactile localization. When the arm is moving, the tactile input in the brain needs to be combined with dynamic information (i.e., proprioceptive feedback or efferent motor commands) about the ongoing arm movement to provide veridical spatial information in external coordinates. The present report addresses how the brain combines these signals in dynamic tactile localization.

The few previous studies on dynamic tactile localization have shown that subjects systematically misperceive the location of tactile stimuli presented near the time of an arm movement (Dassonville 1995; Maij et al. 2011b; Watanabe et al. 2009). Until now, however, few explanations for these tactile localization errors have been provided. For example, Dassonville (1995) suggested that the localization errors relate

Address for reprint requests and other correspondence: F. Maij, Radboud Univ. Nijmegen, Donders Inst. for Brain, Cognition and Behaviour, Montessorilaan 3, 6525 HR Nijmegen, The Netherlands (e-mail: f.maij@donders.ru.nl). to a temporal delay in the perception of the tactile stimulus, probably due to a combination of somatosensory delays and the internal misrepresentation of the movement trajectory. But how such delays cause the subsequent misrepresentation in a biological system is not straightforward, and requires a modeling approach. Here we consider the effects of temporal delay as well as temporal and spatial variability that characterizes biological systems, testing the hypothesis that tactile mislocalization is the consequence of the brain's attempt to integrate sensory and motor signals in a statistically optimal fashion.

Support for this hypothesis originates from analogous findings reported in the visual literature. That is, human participants make systematic errors when localizing objects flashed around the time of saccades (Honda 1989; Lappe et al. 2000; Maij et al. 2009; Mateeff 1978; Matin and Pearce 1965; Ross et al. 1997; Schlag and Schlag-Rey 1995). While previous models suggest that these localization errors arise from the temporal low-pass filtering properties of retinal (Pola 2004, 2007) or extraretinal (Dassonville et al. 1992) processing, a more recent account suggests that temporal uncertainty in combining information about the object's retinal location and information about eye position is responsible for the mislocalization effect (Maij et al. 2011a; Maij et al., unpublished observations). Would this account of temporal uncertainty generalize to errors in dynamic tactile localization?

One of the predictions of the optimal integration model, explained in more detail below, is that localization errors depend on movement velocity. While this prediction cannot easily be tested with saccades because of their fixed amplitude-velocity relationship (Collewijn et al. 1988), it is interesting to test this in the context of arm movements for which velocity is under voluntary control. In the present study, we therefore examined the role of arm velocity in tactile localization, testing how well the optimal integration model explains these findings.

\section{METHODS}

Nine participants gave informed consent to take part in the experiment. All subjects had normal or corrected-to-normal visual acuity and were free of any known sensory, perceptual, or motor disorders. One participant was excluded because she had difficulty in performing the arm movements at the correct velocity. The remaining participants (5 men, 3 women; 20-30 yr old) made movements with the extended right arm. The study was part of a research program approved by the ethics committee of the Social Sciences Faculty of the Radboud University in Nijmegen.

Setup. Participants were seated in complete darkness. They were tested with their torso parallel to a screen placed $90 \mathrm{~cm}$ in front of them. Two LEDs placed on the screen at shoulder level served as 
targets for pointing (see Fig. 1). The pointing targets were arranged around the arm's straight-ahead pointing position, with a distance of $80 \mathrm{~cm}$ between them.

A vibrotactile device ( $30 \mathrm{~g}$; Haptuator, Tactile Labs) was attached to the tip of the right index finger. A small pin attached to the membrane of the tactile device was presented as a pulse of $5 \mathrm{~ms}$ (half a sine wave with a frequency of $100 \mathrm{~Hz}$ ) with an acceleration of $\sim 1.1$ $g$. The Haptuator was oriented orthogonally to the movement path of the arm. The position of the right index finger in space was recorded with an Optotrak system (Northern Digital, Waterloo, ON, Canada; sampling rate $=500 \mathrm{~Hz}$, accuracy $<0.1 \mathrm{~mm}$ ). A data acquisition unit (Odau, Northern Digital; sampling rate $=500 \mathrm{~Hz}$ ) was used to synchronize the position data with the timing of the tactile stimulus. The experiment was controlled within MATLAB (MathWorks, Natick, MA) using the Optotrak Toolbox (http://webapp6.rrz.uni-hamburg.de/allpsy/vf/ OptotrakToolbox). The tactile stimulus, driven with waveforms as though it were a common loudspeaker, was programmed with the Psychophysics Toolbox (Brainard 1997).

Procedure. A trial began with the participant pointing toward one of the two LEDs (presented in random order), illuminated for a uniformly distributed random time interval of $1.5-1.75 \mathrm{~s}$. Subsequently, the other LED was illuminated for $50 \mathrm{~ms}$, indicating that the participant should point toward it with the right arm at either a fast speed $(1.25-2.5 \mathrm{~m} / \mathrm{s})$ or a slower speed $(0.63-0.83 \mathrm{~m} / \mathrm{s})$. At a random time before, during, or after the movement a tactile stimulus was delivered. After the arm movement had finished, the participant had to indicate the perceived location of the tactile stimulus by pointing at it with the same hand that made the arm movement and pressing a button with the other hand when done (see Fig. $1 B$ ). Next, auditory feedback was provided to specify whether the arm movement was made within the required speed limits. The two velocity conditions were presented in four alternating blocks of 75 trials each, separated by a brief rest. Practice trials were included before the experiment started until the participant felt confident with the task.

Data analysis. Data were analyzed with MATLAB software. We used the recorded positions of the index finger at the time of the button press to determine the localization error (see Fig. 1B). Positive values of the localization error indicate an error in the direction of the arm movement target (i.e., indicating a position that is too far to the right for a rightward movement). We plotted the localization error as a function of the different locations and times of the tactile stimulus (relative to movement onset).

Start and end of the movement were determined based on a velocity threshold of $5 \mathrm{~cm} / \mathrm{s}$. We discarded trials in which the participant started moving too early, before the second LED turned on. We also discarded trials in which the arm displacement was shorter than $35 \mathrm{~cm}$.

Optimal integration model. We developed a tactile localization model, following an approach similar to that proposed for perisaccadic mislocalization of briefly presented visual stimuli (Maij et al.

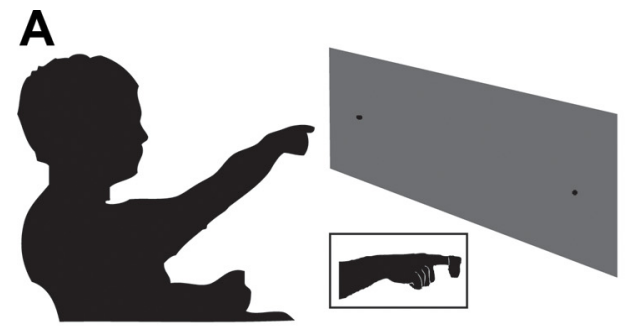

2011a; Maij et al., unpublished observations). Figure $2 A$ shows a graphical description of the model, which assumes statistically optimal processing of the sensory signals involved, including the tactile receptors and arm position signals.

To determine the location at which the stimulus was presented while making an arm movement the observer needs to know the time of the stimulus and the position of the arm at the time of the stimulus. Let us, for simplicity, first consider the situation in which there is no uncertainty about the time of the stimulus and the position of the arm. In this case, the exact location of the stimulus in external space $x_{\text {ext }}$ is given by $x_{\text {ext }}=x_{\text {arm }}\left(t_{\text {stim }}\right)$, in which $x_{\text {arm }}$ depicts the arm position at time $t_{\text {stim }}$ of the stimulus.

When taking into account the noise about the time of the stimulus and the noise about the position of the arm at the time of the stimulus, we need to translate the equation into a statistical framework. First, we assume that uncertainty about the time of the stimulus $\left(p_{t_{\text {stim }}}\right)$ is a normal distribution $(N)$ around the time of the stimulus $\left(t_{\text {stim }}+d\right)$ with standard deviation $\sigma_{t}$. The hat denotes an observation.

$$
p_{t_{\text {stim }}}\left(\hat{t}_{\text {stim }} \mid t_{\text {stim }}\right)=N\left(t_{\text {stim }}+d, \sigma_{t}\right)
$$

The delay $(d)$ represents a mismatch in the perceived time of the stimulus with respect to the time of the arm movement. A positive value for $d$ indicates that the participant's internal estimate of arm position is $d \mathrm{~ms}$ ahead of its actual position at the time of the stimulus; a negative value for $d$ indicates that the participant's internal representation of arm position lags its actual position at the time of the stimulus. The standard deviation $\left(\sigma_{t}\right)$ represents temporal uncertainty about the time of the stimulus.

To derive the probability distribution of $x_{\text {arm }}$, which is a function of $t_{\text {stim }}$, we apply the Jacobian transformation $|P(x) \mathrm{d} x|=|P(t) \mathrm{d} t|$ to the probability distribution of $p_{t_{\text {stim }}}$,

$$
p_{x_{\text {arm }}}\left(x\left(\hat{t}_{\text {stim }}\right) \mid t_{\text {stim }}\right)=p_{t_{\text {stim }}}\left(\hat{t}_{\text {stim }}\left(x_{\text {arm }}\right) \mid t_{\text {stim }}\right)\left|\frac{\mathrm{d} x_{\text {arm }}(t)}{\mathrm{d} t}\right|^{-1}
$$

where $t_{\text {stim }}\left(x_{\text {arm }}\right)$ represents the inverse of the arm trajectory, that is, time as a function of the arm position. In other words, the probability of the position of the arm position given the uncertainty of $t_{\text {stim }}$ depends not only on the probability distribution of $t_{\text {stim }}$ but also on arm velocity. Arm velocity is the variable that is modulated in the present study.

It is further assumed that the internal estimate of arm position based on proprioception is contaminated with noise, which is here also represented as a normal distribution,

$$
p_{x_{\text {arm }}}\left(x \mid \hat{x}_{\text {arm }}\left(\hat{t}_{\text {stim }}\right)\right)=N\left(x_{\text {arm }}\left(\hat{t}_{\text {stim }}\right), \sigma_{\text {arm }}\right)
$$

with mean $x_{\text {arm }}\left(t_{\text {stim }}\right)$ and standard deviation $\sigma_{\text {arm }}$. Note that the noise level in the arm position estimates is probably small compared with

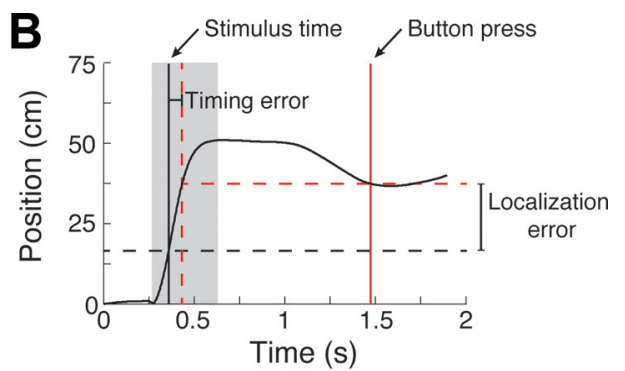

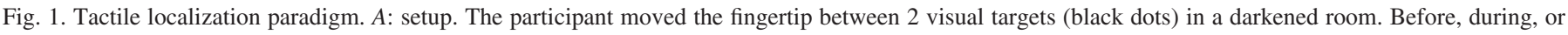

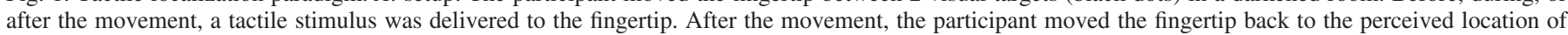

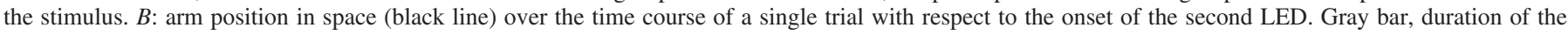

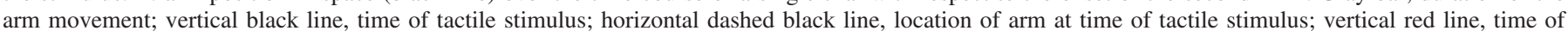

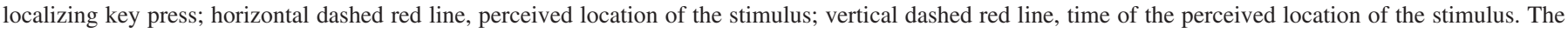

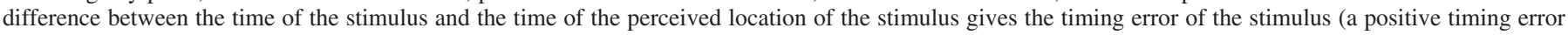
is shown here). 

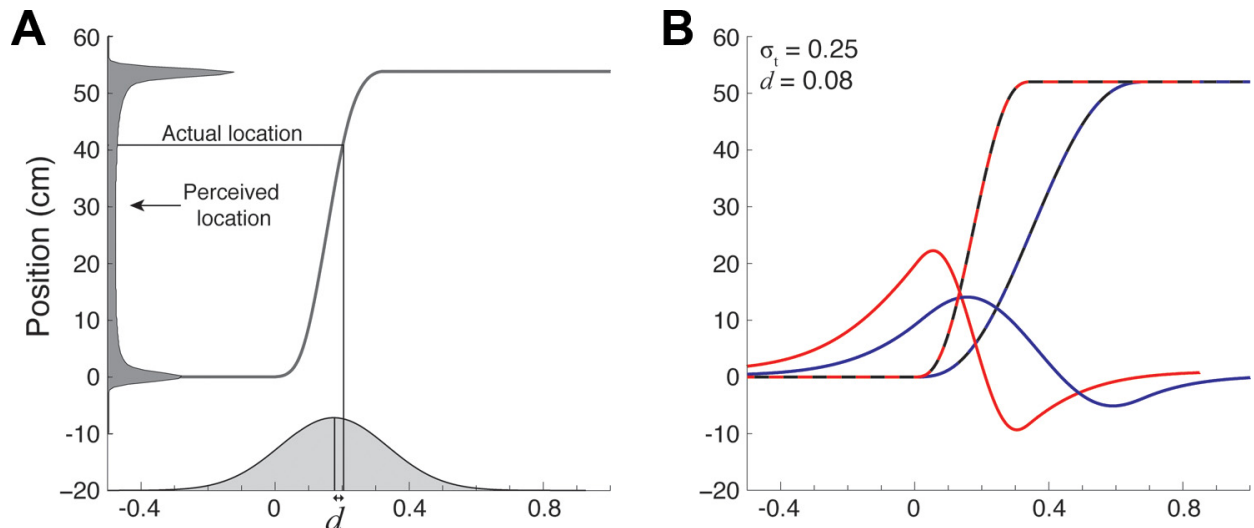

Fig. 2. Graphical description of the model and simulations. A: single-trial prediction for 1 (data) trial of 1 participant. Uncertainty with a delay in the temporal processing of the tactile stimulus (gray Gaussian at $204 \mathrm{~ms}, \sigma$ is $156 \mathrm{~ms}$, delay is $-26 \mathrm{~ms}$ for this particular participant) is combined with the arm movement (amplitude of $52 \mathrm{~cm}$ and duration of 350 ms; dark gray line). The resulting posterior distribution is represented at the $y$-axis (gray curve). The mean of this posterior distribution represents the perceived location (indicated by arrow) of the stimulus, whereas the actual location of the hand is further toward
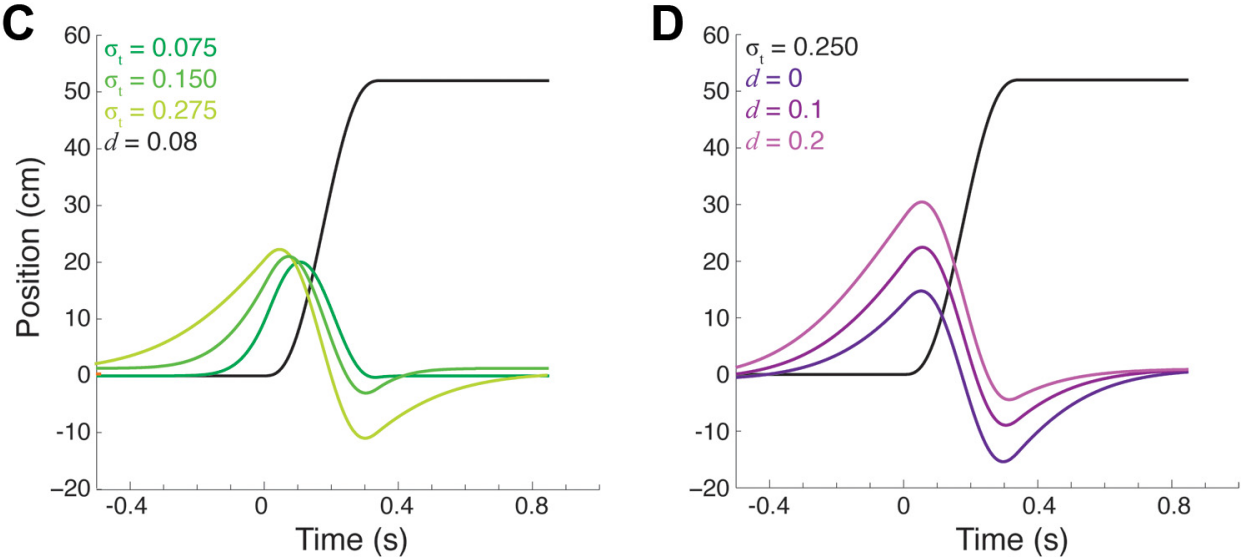
the end point of the arm movement (indicated by horizontal black line). $B$ : predicted localization error patterns for arm movements of $52 \mathrm{~cm}$ for 2 different arm movement durations (blue, $0.7 \mathrm{~s}$; red, $0.35 \mathrm{~s}$ ). $C$ and $D$ : predicted localization error patterns for arm movements of $52 \mathrm{~cm}$ in $0.35 \mathrm{~s}$. Different widths of temporal uncertainty $\left(\sigma_{t} ; C\right)$ and delays $(d ; D)$ result in different localization patterns.

the errors of mislocalization, considering results from van Beers et al. (1998), who reported the precision of proprioceptive localization of the arm $<1 \mathrm{~cm}$.

The posterior probability density distribution of the arm at the time of stimulus presentation of $x_{\text {ext }}$ is given by the distribution that results from the multiplication of the distributions of the uncertainty of the time of the stimulus and the noise in arm position:

$$
\begin{aligned}
& p_{x_{\text {arm }}}\left(\hat{x}_{\text {arm }}\left(\hat{t}_{\text {stim }}\right) \mid t_{\text {stim }}, \hat{x}_{\text {arm }}\left(\hat{t}_{\text {stim }}\right)\right) \\
& =\int P_{x_{\text {arm }}}\left(\hat{x}_{\text {arm }} \mid x_{\text {arm }}\left(\hat{t}_{\text {stim }}\right)\right) p_{t_{\text {stim }}}\left(\hat{t}_{\text {stim }}\left(x_{\text {arm }}\right) \mid t_{\text {stim }}\right)\left|\frac{\mathrm{d} x_{\text {arm }}(t)}{\mathrm{d} t}\right|^{-1} \mathrm{~d} x_{\text {arm }}
\end{aligned}
$$

The mean of this distribution $(E q .4)$ is taken as the participant's estimate of the location of the stimulus in external space (see Fig. 2A) (Knill and Pouget 2004; Roach et al. 2006; Saunders and Knill 2001). Note that taking the arm position with the highest probability, i.e., the maximum a posteriori (MAP), as the model prediction of the external location of the stimulus is not reasonable, because the distribution is not normally distributed.

We note that in the model derivation presented above we explicitly isolated arm velocity, as represented by the Jacobian (Eq. 2), by taking the integral over position (Eq. 4). However, the same model can also be formulated differently, without an explicit representation of velocity, using the law of probability by taking the integral over time instead of position.

In this model we only consider temporal uncertainty about the time of the stimulus and the proprioceptive noise about the position of the arm at the time of the stimulus as crucial elements of the model. We note that there could also be temporal uncertainty in the internal representation of the arm. Yet, in modeling terms, this factor cannot be distinguished from the stimulus uncertainty. Moreover, such noise contribution may also result in the spatial uncertainty of the hand trajectory. Obviously, several other noise sources can be included in the model, but we explicitly chose not to add them, to clearly demonstrate that the temporal uncertainty about the time of the stimulus is a major factor in the explanation of the observed tactile mislocalization patterns. For further elaboration on how to deal with several noise sources, we refer to the perisaccadic visual mislocalization model (Maij et al., unpublished observations).

To facilitate the interpretation of the data, we now evaluate the predictions of this model for various parameter values.

Model simulations. We first determined the model's predictions of the localization pattern by varying the two free parameters of the model (delay $d$ and width of the temporal uncertainty $\sigma_{t}$; see Fig. 2, $C$ and $D$ ). Subsequently, we made predictions for the localization errors for two different arm velocities (Fig. 2B). To simulate arm position as a function of time we used a minimum jerk trajectory (Flash and Hogan 1985),

$x_{\mathrm{arm}}(t)=x_{\mathrm{i}}+\left(x_{f}+x_{\mathrm{i}}\right)\left(10(t / a)^{3}-15(t / a)^{4}+6(t / a)^{5}\right)$

for arm movements from an initial location $x_{i}$ to the final location $x_{f}$ in $a \mathrm{~s}$. Note that with the inverse of the derivative of the minimum jerk trajectory, i.e., a bell-shaped arm velocity profile, Eq. 4 cannot be expressed in analytical terms but requires a numerical approximation. We further set the value of the precision of proprioceptive localization of the arm to $0.7 \mathrm{~cm}$ (van Beers et al. 1998), although the value of this parameter has only a negligible effect on the predicted localization patterns (not shown). Figure 2 presents the model simulation for arm movements of $52 \mathrm{~cm}$ for a single trial (Fig. $2 A$ ) and multiple trials (Fig. 2, $B-D$ ). We varied arm movement duration (Fig. $2 B$ ), the width of the temporal uncertainty (Fig. 2C), and the delay parameter (Fig. $2 D)$. As shown, small differences of the free parameters $\left(\sigma_{t}\right.$ and $\left.d\right)$ result in large differences in the predicted localization error patterns.

Model fitting. In RESULTS we compare the model with actual data. In fitting the model we used the actual arm kinematics, not the minimum jerk trajectory. The model contains two free parameters $\left(\sigma_{t}\right.$ and $\left.d\right)$ that were fitted to all data for each subject. Proprioceptive variance of arm 
position was again set to $0.7 \mathrm{~cm}$ (van Beers et al. 1998). We fitted the model by maximizing the likelihood of $\sigma_{\mathrm{t}}$ and $d$, given the data (maximum likelihood estimation, MLE). Optimal parameter values were obtained by minimizing the negative log-likelihood function.

In a further step, we reduced the model to a one-free parameter model, represented by $\sigma_{t}$. We removed $d$ as a free parameter from the model by setting it to a fixed parameter value determined from the data as a timing error, following Dassonville (1995). In his study he calculated the timing error as the difference between the time of the stimulus and the time at which the finger was at the location that was indicated as the perceived location of stimulus (see Fig. 1; Dassonville 1995). Here we use this method to calculate the delay parameter $d$ for each participant. More specifically, $d$ was determined by taking the average of the timing errors of all trials in which the stimulus occurred during the arm movement.

We used a log-likelihood ratio test to compare the two- and one-parameter versions of the model. A $\chi^{2}$-test with one free parameter (the difference in degrees of freedom between the models) was used to calculate the $P$ value. Furthermore, the Bayesian information criterion (BIC; Schwarz 1978) was used to determine which model provides the best fit to the data. The BIC, which provides a measure of the adequacy of the number of model parameters, is defined as BIC = $-2 \cdot \ln L+k \ln (n)$, where $L$ is the maximized value of the likelihood function for the estimated model, $k$ the number of free parameters, and $n$ the number of data points. A more appropriate model is characterized by a lower BIC value.

\section{RESULTS}

On average, we analyzed $243 \pm 24$ trials (mean \pm SD) across participants. Of these movements, the durations of the fast and slow arm movements were $337 \pm 64 \mathrm{~ms}$ and $579 \pm 87$ ms (mean $\pm \mathrm{SD}$ ), respectively, averaged across participants. Averaged amplitudes of the fast and slow arm movements, $49 \pm 5 \mathrm{~cm}$ and $46 \pm 4 \mathrm{~cm}$ (mean $\pm \mathrm{SD}$ ), respectively, were significantly different (paired $t$-test, $P<0.05$ ). As shown below, this difference in amplitude is substantially smaller than the difference between these conditions in localization errors.

We examined the external localization of a tactile stimulus presented to the fingertip of a slow- or fast-moving arm. If participants were perfect in integrating tactile input and dynamic feedback about the ongoing arm movement, localization errors should be zero. Figure 3 shows the localization errors as a function of the time of the stimulus relative to the onset of the movement, for high- and low-velocity arm movements. The respective panels of Fig. 3, which illustrate the results from each participant, immediately convey the general impression that localization errors are larger for fast compared with slow movement speed, as predicted by our model (see Fig. 2). However, there is some intersubject variability. For instance, participants $S 1$ and $S 3$ produced the largest positive errors (i.e., a spatial shift in the direction of the arm movement) when the tactile stimulus was presented just prior to movement onset, while $S 4$ and $S 5$ demonstrated clear negative errors during the movement. For each participant we fit a moving Gaussian window across the data points (represented by the solid lines in Fig. 3) for each velocity condition separately. The peak-to-peak values of these localization curves (fast mean \pm SD: $23.5 \pm 4.4 \mathrm{~cm}$, slow mean \pm SD: $14.5 \pm 3.0 \mathrm{~cm}$ ) showed that the amplitude was significantly larger for the high-velocity arm movements than for the low-velocity movements (paired $t$-test, $P<0.05)$.

The question is, are these localization patterns consistent with the predictions of our model? We used the observed arm kinematics to model $x_{\text {arm }}$ and predict the localization errors of each participant. For each subject, we determined the two parameters of the model by fitting the $\sigma_{t}$ and $d$ to all data simultaneously, irrespective of arm movement velocity. Figure 4 illustrates the best-fit lines based on the moving averages of the predicted error in each single trial. These indicate that, in qualitative terms, the model provides a good account of the characteristics of the data, revealing the observed differences between fast and slow speeds of movement. For each participant, the best-fit parameter values are listed in Table 1. Across participants, we found a mean $\sigma_{t}$ of $143 \pm 17 \mathrm{~ms}$ (mean $\pm \mathrm{SD}$ ) and a mean delay $d$ of $-1 \pm 46 \mathrm{~ms}$ (mean $\pm \mathrm{SD})$. Note the small standard deviation of $\sigma_{t}$ compared with the standard deviation of $d$.

To further scrutinize the consistency between the measured and modeled localization errors, we normalized both in terms of movement duration. Figure 5 presents the results of this analysis, showing a clear increase in localization errors for the fast velocity condition. In addition, the model also accounts for the differences among the participants. In other words, the fit parameters $\left(\sigma_{t}, d\right)$ of the model in combination with the actual arm movement trajectories provide a very reasonable explanation for the error pattern of each participant.

So far, the model considered has two free parameters $\left(\sigma_{t}, d\right)$ to explain each individual data set. However, as mentioned above, the delay parameter $(d)$ is possibly directly linked to the timing error, which represents the shift in time between the arm movement and the perceived time of the stimulus (Dassonville 1995; see Fig. 1B). Indeed, the average values of the timing error were closely related to fit values of the delay parameter ( $r=0.88, P<0.01)$. Furthermore, calculating the $\chi^{2}$ values for each participant showed that the model with one free parameter was not significantly different from the two-parameter model $(P>>0.05,1$ dof $)$. In fact, on the basis of the BIC, the model with only one free parameter performs better than the model with two free parameters [for all participants BIC values for the 1-free parameter model were smaller (total sum of -67.9 ) than the BIC values for the 2-parameter model (total sum of -22.9$)$ ].

\section{DISCUSSION}

We found that the perceived location of a brief tactile stimulus that was presented before, during, or after the arm movement depends on the velocity of the movement. Localization errors were more pronounced for higher than lower arm velocities. An optimal integration model, involving uncertainty about the time of the stimulus, nicely describes the different localization error patterns. This suggests that spatial errors in dynamic tactile perception stem from the temporal aspects of stimulus processing. In the next sections we relate our results to previous work, make a comparison to studies in the visual domain, and discuss how our model is related to other models.

Comparison with previous work. Only a few studies have investigated tactile localization errors during arm movements. Dassonville (1995) was the first to study tactile localization during arm movements. Interestingly, he reported more pronounced errors than in the present and other related studies (Maij et al. 2011b; Watanabe et al. 2009). One possible reason could be differences in arm velocity. In our study we asked subjects to perform the fast arm movement similarly to the arm velocity in the paradigm that Dassonville used. Whereas Das- 

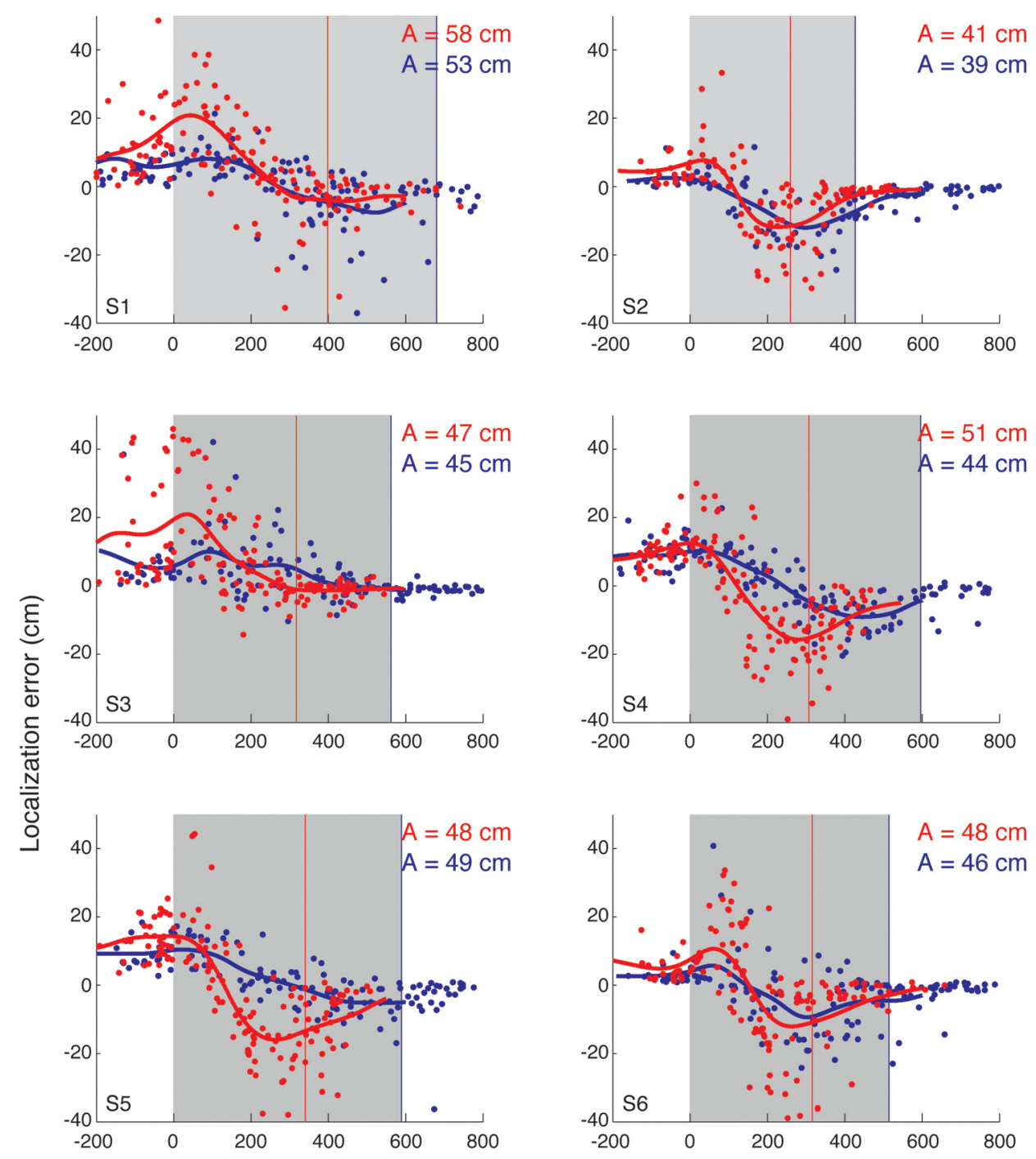

Fig. 3. Localization errors (dots) relative to the onset of the arm movement [with average duration (gray interval) and amplitude A] for the 8 participants $(S 1-S 8)$. Red dots, fast movements; blue dots, slow movements. Solid lines represent a moving Gaussian average window across the data points (width of 100

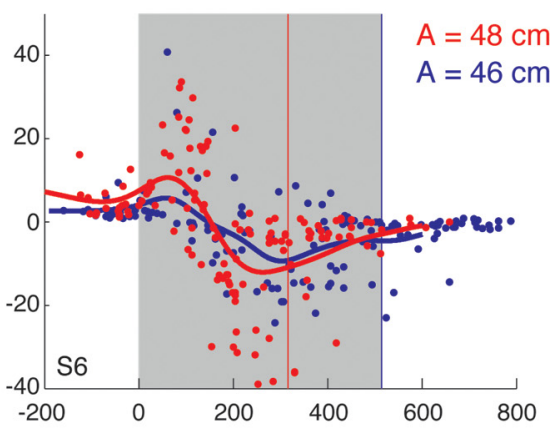
$\mathrm{ms})$. Red solid vertical line represents average movement duration for the fast movements and blue solid vertical line for the slow movements. Because of axis limits, 3 data points are not shown.

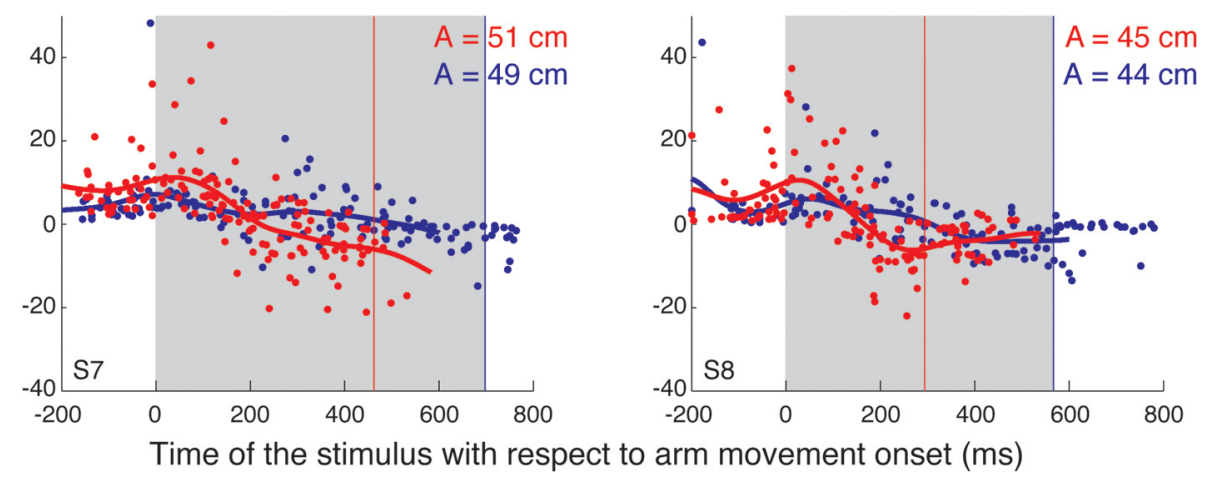

sonville (1995) found large errors in the direction of the movement at movement onset and small errors in the opposite direction of the movement around movement offset, we found large positive and negative localization errors at movement onset and offset for the fast arm movement condition, respectively. Thus these differences between the localization patterns are unlikely to be related to differences in arm velocity.

Could the differences between the localization patterns relate to the type of tactile stimulus? Watanabe and colleagues
(2009) showed that the type of vibrotactile stimuli affects its perceived location during arm movements. They found similar types of localization patterns in responses to a transient vibration $(130 \mathrm{~Hz})$ stimulus of $20-\mathrm{ms}$ duration and the onset of a continuous vibration of $1 \mathrm{~s}$ but a temporal shift in the localization patterns in responses to the onset and offset of continuous 1-s vibrations. These differences between the patterns look remarkably similar to the differences in the localization patterns that we found here and those of Dassonville (1995). 

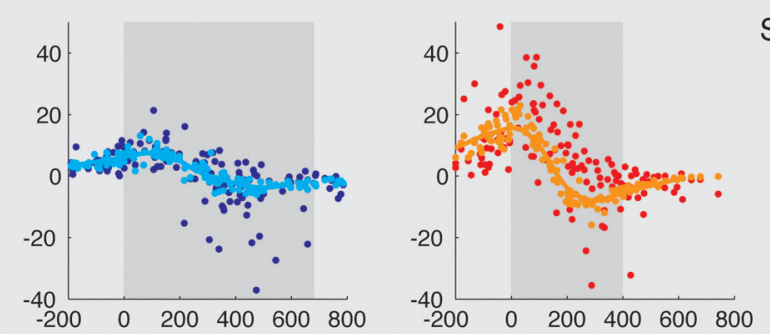

S1
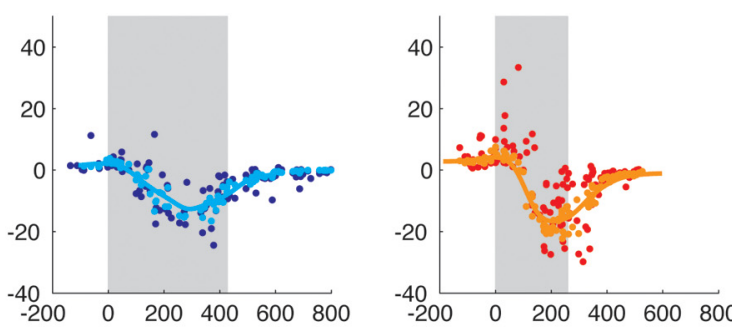

S3
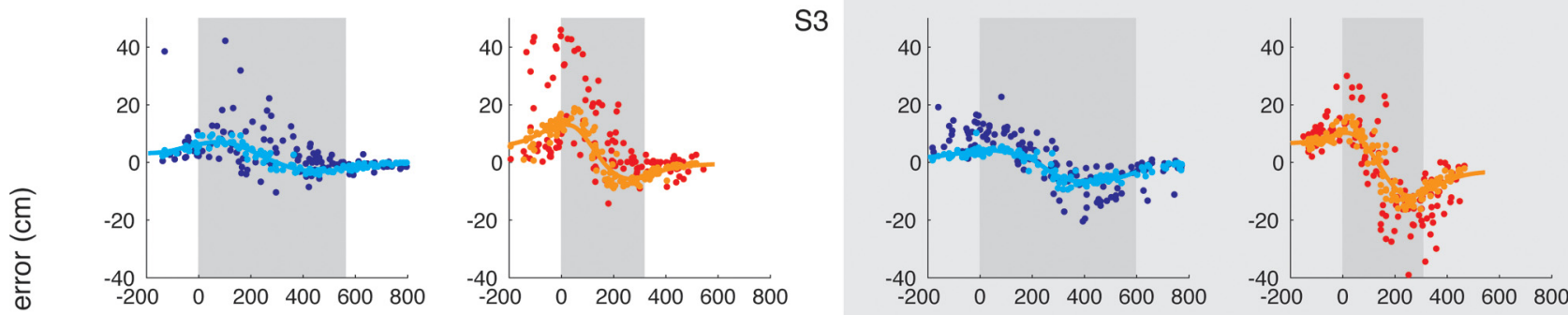

S4

S5
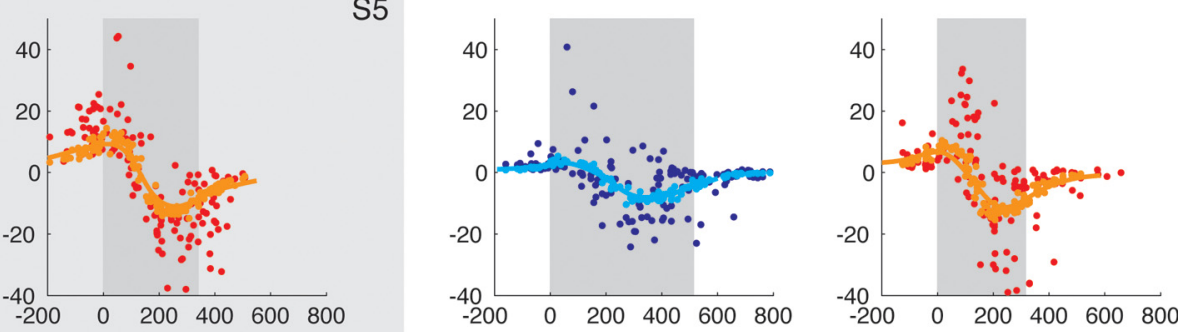

S7
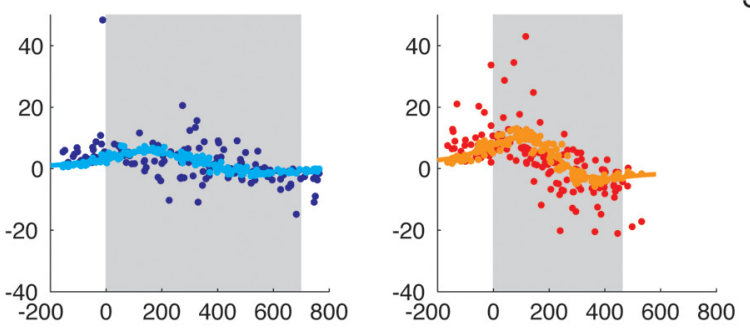

Time of stimulus with respect to the onset of the arm movement (ms)

Fig. 4. Model fits for each single localization trial. For each participant 2 panels are shown for each arm velocity: slow (left) and fast (right). Cyan (slow movements) and orange (fast movements) colored dots depict the predicted perceived locations for each trial. See Fig. 3 for further details and Table 1 for the fit parameters of $\sigma_{t}$ and $d$ for each participant.

However, because our vibrotactile stimuli were even shorter (pulse of $5 \mathrm{~ms}$ ) than the transient stimulus that Watanabe and colleagues used, we consider it also unlikely that the differences between the study of Dassonville (6-ms pulse) and our study as well as the present intersubject differences are due to judging the stimulus based on its onset versus its offset.

It is possible that the localization differences among the various stimuli in the study by Watanabe and colleagues (2009)

Table 1. Best fit parameters for all participants

\begin{tabular}{lrrrrrrrr}
\hline \hline & $S 1$ & $S 2$ & $S 3$ & $S 4$ & $S 5$ & $S 6$ & $S 7$ & $S 8$ \\
\hline$\sigma_{t}, \mathrm{~ms}$ & 179 & 130 & 156 & 160 & 158 & 134 & 132 & 148 \\
$d, \mathrm{~ms}$ & 13 & -97 & 24 & -36 & -38 & -43 & 32 & -19
\end{tabular}

$\sigma_{t}$, Standard deviation of time of stimulus; $d$, delay parameter. are due to different timing properties. In analogy with saccades, it was recently shown that presenting an irrelevant tone around the time of the flash resulted in a temporal shift of the localization pattern (Maij et al. 2009). To explain these results, it was suggested that the flash and the tone are optimally integrated, but only if both were presented closely enough in time. A similar mechanism could also explain the results of Watanabe and colleagues (2009), which would be an 0interesting research topic for future work.

A further factor that could influence tactile localization is the vibration frequency of the stimulus. It has been shown that a vibrotactile stimulus is perceived more intensely if vibration frequency is higher (Bensmaia and Hollins 2003; Klatzky and Lederman 1999). However, here we kept stimulus frequency invariant. Furthermore, it has been reported that perceptual thresholds of tactile stimuli are higher during movement com- 

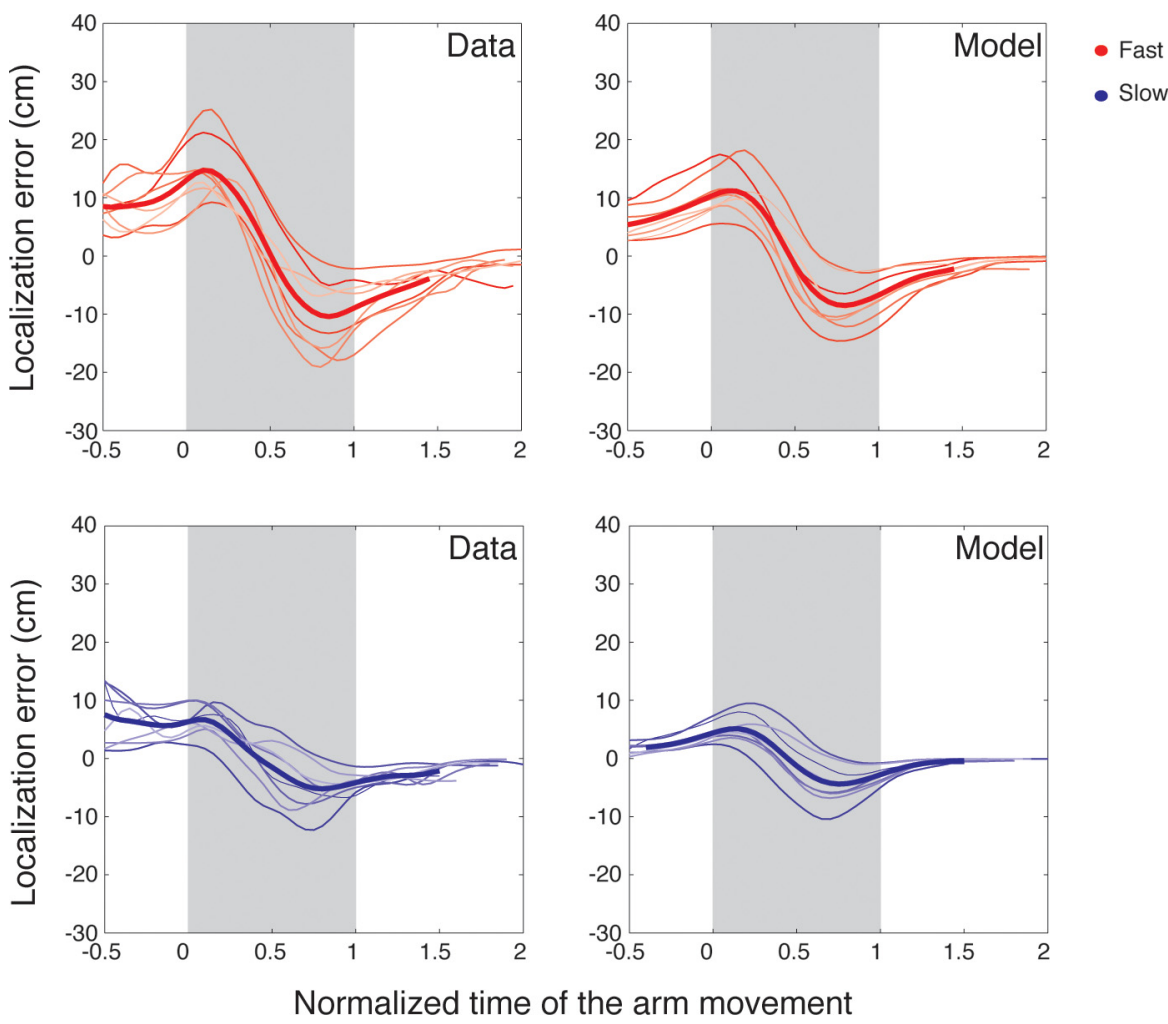

Fig. 5. Tactile localization patterns for fast (red) and slow (blue) arm movements: data vs. model. Data and model curves are color matched for each participant. Movement duration was normalized (gray bar). Thick lines represent averages across participants. pared with rest (Juravle et al. 2010). In our experiment, stimuli were always well above threshold, so it is not likely that this factor played a role in the present experiment.

Model evaluation. Our model assumes that the perceived external location of the tactile stimulus is a function of arm position at the time of the stimulus. Because there is temporal uncertainty and delay in the internal processing of the stimulus, arm position at the time of the stimulus also becomes a stochastic variable. The temporal uncertainty propagates into the perceived location of the stimulus, depending on arm velocity (see Eq. 4). It is a straightforward assumption that stimulus processing is noisy and delayed, which holds for any biological system. The temporal precision of neural coding typically depends on the attribute of the stimulus [e.g., contrast in vision (Victor and Purpura 1996) or stimulus frequency in vision (Nowak et al. 1997) and in touch (Mackevicius et al. 2012)].

The model was fit to the results of each individual, simultaneously with data of both velocity conditions. Fits of the data showed that the model explains the results of both velocity conditions. Fit parameters ranged between 130 and $179 \mathrm{~ms}$ for the width of temporal uncertainty and between -97 and $32 \mathrm{~ms}$ for the delay parameter. In fact, the only crucial variable in the model seems to be temporal uncertainty; the delay parameter could be approximated by the timing error in the data.

Could our model also explain the findings of tactile localization previously observed by Dassonville (1995)? Dassonville found a large positive localization error around movement onset and almost no negative error around movement offset of the arm. To simulate these localization patterns, the present model not only requires a smaller temporal uncertainty ( $\sigma$ is $\sim 75 \mathrm{~ms}$ ) than we found here, it also requires a value for the time delay of $\sim 80 \mathrm{~ms}$. The latter is in close correspondence with Dassonville (1995), who calculated a timing error of 88 $\mathrm{ms}$, averaged across participants. Thus our model not only explains our own data set, obtained with two arm velocity conditions, it also nicely captures the observations by Dassonville (1995), obtained in a completely independent manner.

Comparison with perisaccadic localization studies and models. It is known that when flashes are presented near the time of a saccade people systematically misperceive the location of the flash (e.g., Honda 1989; Lappe et al. 2000; Maij et al. 2009; Mateeff 1978; Matin and Pearce 1965; Ross et al. 1997; Schlag and Schlag-Rey 1995). There are, however, conflicting results regarding the effect of saccade velocity on such perisaccadic localization errors. While some studies showed a systematic effect of saccade velocity (Ostendorf et al. 2007), others did not (Maij et al. 2012). A possible explanation is found in the fixed amplitude-velocity relationship for saccadic eye movements (Collewijn et al. 1988). This relationship implies that velocity cannot be manipulated without changing amplitude. Additionally, it has been found that localization errors increase with saccade amplitude (Lavergne et al. 2010).

We suggest that haptic localization errors and perisaccadic localization errors point to a similar mechanism: temporal uncertainty about the time of the stimulus with respect to the movement. The conflicting results found in the saccadic literature (Maij et al. 2012; Ostendorf et al. 2007) might therefore be best tested in the haptic modality since, in contrast to saccade velocity, arm velocity is under voluntary control. Arm velocity can thus be modulated irrespective of amplitude, although Fitts's law suggests that faster velocity goes at the expense of accuracy. Our participants performed the fast arm movements with the maximum velocity they could achieve. As 
a result, we found that the localization errors are larger for the fast velocity condition than the slow velocity condition.

Possible models of perisaccadic perception suggest that localization errors arise from the temporal low-pass characteristics of the retinal (Pola 2004, 2007) or the extraretinal (Dassonville et al. 1992) signals. However, these models are not able to explain all observations, e.g., the compression of the perceived location of a flash toward the eye position (or saccade target). Other models suggest a remapping of receptive fields (Ross et al. 2001) or a shift in spatial attention toward the saccade target (Hamker 2005; Hamker et al. 2008). These models are able to explain compression effects but not the localization biases independently of flash location. Indeed, a recent model that can explain both compression and biases is a temporal uncertainty model (Maij et al. 2011a; Maij et al., unpublished observations), which also provides the basis of the present tactile localization model.

Note that the perisaccadic localization model by Maij et al. (2011a; unpublished observations) also includes a foveal bias, to represent the brain's assumption that if flashes are perceived then they must have occurred close to the fovea (Brenner et al. 2006, 2008). Maij et al. (2011a; unpublished observations) modeled this foveal bias as an a priori distribution centered on the direction of gaze at the moment of the flash, resulting in the flash being attracted to the fovea, i.e., a compression toward eye position (e.g., Lappe et al. 2000; Ross et al. 1997). In the tactile localization model we assume the prior distribution to be flat. Because the Haptuator was attached to the index finger, and therefore delivered the stimulus always at the same position on the hand, there is no reason to assume a spatial bias. In future work, it would be interesting to present tactile stimuli at different locations on the body to test whether the brain makes use of similar somatotopic biases as in retinal processing.

\section{ACKNOWLEDGMENTS}

We thank Ivar Clemens, Pascal de Water, and Hubert Voogd for technical support, Dr. Mathieu Koppen for discussion about the modeling aspects, Thomas van der Kraan for pilot studies, and Dr. Luc Selen for comments on an earlier version of the paper.

\section{GRANTS}

This research was supported by the Netherlands Organization for Scientific Research (NWO, Rubicon Grant 446-10-015 and Veni Grant 451-12-009) to F. Maij, the European Research Council under the European Union's Seventh Framework Programme (FP7/2007-2012)/ERC Grant agreement (no 283567) to W. P. Medendorp, the NWO (400-07-003) to W. P. Medendorp, and EU FP7 ICT grant CogLaboration (no. 287888) to A. M. Wing.

\section{DISCLOSURES}

No conflicts of interest, financial or otherwise, are declared by the author(s).

\section{AUTHOR CONTRIBUTIONS}

Author contributions: F.M., A.M.W., and W.P.M. conception and design of research; F.M. performed experiments; F.M. analyzed data; F.M., A.M.W., and W.P.M. interpreted results of experiments; F.M. prepared figures; F.M. drafted manuscript; F.M., A.M.W., and W.P.M. edited and revised manuscript; F.M., A.M.W., and W.P.M. approved final version of manuscript.

\section{REFERENCES}

Azanon E, Soto-Faraco S. Changing reference frames during the encoding of tactile events. Curr Biol 18: 1044-1049, 2008.
Bensmaia SJ, Hollins M. The vibrations of texture. Somatosens Motor Res 20: 33-43, 2003.

Brainard DH. The Psychophysics Toolbox. Spat Vis 10: 433-436, 1997.

Brenner E, Mamassian P, Smeets JB. If I saw it, it probably wasn't far from where I was looking. $J$ Vis 8: 7.1-7.10, 2008.

Brenner E, van Beers RJ, Rotman G, Smeets JB. The role of uncertainty in the systematic spatial mislocalization of moving objects. J Exp Psychol Hum Percept Perform 32: 811-825, 2006.

Collewijn H, Erkelens CJ, Steinman RM. Binocular co-ordination of human horizontal saccadic eye movements. J Physiol 404: 157-182, 1988.

Dassonville P. Haptic localization and the internal representation of the hand in space. Exp Brain Res 106: 434-448, 1995.

Dassonville P, Schlag J, Schlag-Rey M. Oculomotor localization relies on a damped representation of saccadic eye displacement in human and nonhuman primates. Vis Neurosci 9: 261-269, 1992.

Flash T, Hogan N. The coordination of arm movements: an experimentally confirmed mathematical model. J Neurosci 5: 1688-1703, 1985.

Hamker FH. The reentry hypothesis: the putative interaction of the frontal eye field, ventrolateral prefrontal cortex, and areas V4, IT for attention and eye movement. Cereb Cortex 15: 431-447, 2005.

Hamker FH, Zirnsak M, Calow D, Lappe M. The peri-saccadic perception of objects and space. PLoS Comput Biol 4: e31, 2008.

Heed T, Roder B. Common anatomical and external coding for hands and feet in tactile attention: evidence from event-related potentials. J Cogn Neurosci 22: 184-202, 2010.

Honda H. Perceptual localization of visual stimuli flashed during saccades. Percept Psychophys 45: 162-174, 1989.

Juravle G, Deubel H, Tan HZ, Spence C. Changes in tactile sensitivity over the time-course of a goal-directed movement. Behav Brain Res 208: 391$401,2010$.

Klatzky RL, Lederman SJ. Tactile roughness perception with a rigid link interposed between skin and surface. Percept Psychophys 61: 591-607, 1999.

Knill DC, Pouget A. The Bayesian brain: the role of uncertainty in neural coding and computation. Trends Neurosci 27: 712-719, 2004.

Lappe M, Awater H, Krekelberg B. Postsaccadic visual references generate presaccadic compression of space. Nature 403: 892-895, 2000.

Lavergne L, Vergilino-Perez D, Lappe M, Dore-Mazars K. The spatial pattern of peri-saccadic compression for small saccades. J Vis 10: 17, 2010.

Mackevicius EL, Best MD, Saal HP, Bensmaia SJ. Millisecond precision spike timing shapes tactile perception. J Neurosci 32: 15309-15317, 2012.

Maij F, Brenner E, Smeets JB. Temporal information can influence spatial localization. J Neurophysiol 102: 490-495, 2009.

Maij F, Brenner E, Smeets JB. Temporal uncertainty separates flashes from their background during saccades. J Neurosci 31: 3709-3711, 2011a.

Maij F, de Grave DD, Brenner E, Smeets JB. Misjudging where you felt a light switch in a dark room. Exp Brain Res 213: 223-227, 2011b.

Maij F, Matziridi M, Smeets JB, Brenner E. Luminance contrast in the background makes flashes harder to detect during saccades. Vision Res 60C: 22-27, 2012.

Mateeff S. Saccadic eye movements and localization of visual stimuli. Percept Psychophys 24: 215-224, 1978.

Matin L, Pearce DG. Visual perception of direction for stimuli flashed during voluntary saccadic eye movements. Science 148: 1485-1488, 1965.

Nowak LG, Sanchez-Vives MV, McCormick DA. Influence of low and high frequency inputs on spike timing in visual cortical neurons. Cereb Cortex 7: 487-501, 1997.

Ostendorf F, Fischer C, Finke C, Ploner CJ. Perisaccadic compression correlates with saccadic peak velocity: differential association of eye movement dynamics with perceptual mislocalization patterns. J Neurosci 27: 7559-7563, 2007.

Pola J. Models of the mechanism underlying perceived location of a perisaccadic flash. Vision Res 44: 2799-2813, 2004.

Pola J. A model of the mechanism for the perceived location of a single flash and two successive flashes presented around the time of a saccade. Vision Res 47: 2798-2813, 2007.

Roach NW, Heron J, McGraw PV. Resolving multisensory conflict: a strategy for balancing the costs and benefits of audio-visual integration. Proc Biol Sci 273: 2159-2168, 2006.

Ross J, Morrone MC, Burr DC. Compression of visual space before saccades. Nature 386: 598-601, 1997. 
Ross J, Morrone MC, Goldberg ME, Burr DC. Changes in visual perception at the time of saccades. Trends Neurosci 24: 113-121, 2001.

Saunders JA, Knill DC. Perception of 3D surface orientation from skew symmetry. Vision Res 41: 3163-3183, 2001.

Schlag J, Schlag-Rey M. Illusory localization of stimuli flashed in the dark before saccades. Vision Res 35: 2347-2357, 1995.

Schwarz G. Estimating the dimension of a model. Ann Stat 6: 461-464, 1978. van Beers RJ, Sittig AC, Denier van der Gon JJ. The precision of proprioceptive position sense. Exp Brain Res 122: 367-377, 1998.

Victor JD, Purpura KP. Nature and precision of temporal coding in visual cortex: a metric-space analysis. J Neurophysiol 76: 1310-1326, 1996.

Watanabe J, Nakatani M, Ando H, Tachi S. Haptic localizations for onset and offset of vibro-tactile stimuli are dissociated. Exp Brain Res 193: 483-489, 2009.

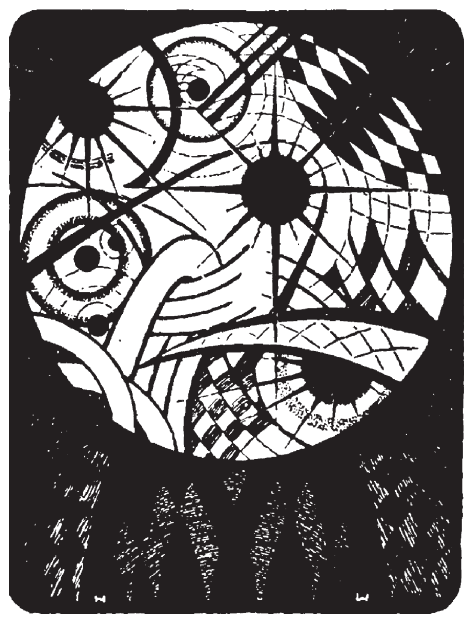

